\title{
Evaluation of the Antioxidant Activities and Tyrosinase Inhibitory Property from Mycelium Culture Extracts
}

\author{
Ki Moon Park, ${ }^{1}$ Kyung Min Kwon, ${ }^{1}$ and Seung Ho Lee ${ }^{2}$ \\ ${ }^{1}$ Department of Food Science and Biotechnology, Sungkyunkwan University, Suwon 440-746, Republic of Korea \\ ${ }^{2}$ Major of Nano-Bioengineering, Incheon National University, Incheon 406-772, Republic of Korea \\ Correspondence should be addressed to Seung Ho Lee; seungho@inu.ac.kr
}

Received 2 June 2015; Revised 14 July 2015; Accepted 16 July 2015

Academic Editor: Yoshiji Ohta

Copyright (c) $2015 \mathrm{Ki}$ Moon Park et al. This is an open access article distributed under the Creative Commons Attribution License, which permits unrestricted use, distribution, and reproduction in any medium, provided the original work is properly cited.

\begin{abstract}
Since mushrooms have many bioactive components, they have been used as components in folk medicine. Because mycelium has an advantage when it comes to large-scale production, this study aimed to evaluate the antioxidant properties and antityrosinase activity from 55 mycelia in culture media. Relatively high 2,2-diphenyl-1-picrylhydrazyl (DPPH) scavenging capacity was detected from the ethanol extract of culture media including mycelium (EECiM) of Morchella esculenta var. esculenta (MEVE), Auricularia polytricha (APO), Tremella aurantia (TAU), Volvariella bombycina (VBO), and Oudemansiella sp. (Osp), which also showed strong reducing power and inhibitory activity in relation to the thiobarbituric acid (TBA) value. On the other hand, relatively high tyrosinase inhibitory activity was detected in Inonotus mikadoi (IMI), Coriolus versicolor (CVE), Volvariella volvacea (VVO), Panellus serotinus (PSE), Auricularia auricula (AAU), and Fomitopsis sp. (Fsp). Interestingly, the APO EECiM exhibited the highest DPPH radical scavenging rate $(77.5 \pm 4.3 \%)$ and reducing power $(1.18 \pm 0.041)$, while the highest inhibitory power of the TBA value and antityrosinase activity were detected in that of TAU $(64.5 \pm 4.1 \%)$ and IMI (46.0 $\pm 7.5 \%)$, respectively. Overall, our study suggested potential candidates for EECiMs that exhibited powerful antioxidant and tyrosinase inhibitory properties and might be used as natural antioxidant tyrosinase inhibitor.
\end{abstract}

\section{Introduction}

Antioxidants are able to inhibit the oxidation of biomolecules by reducing the reactive oxygen species (ROS) level to prevent the damage caused by free radicals in body cells. Free radicals destructively react with biomolecules, inducing diseases such as cancer [1], aging [2], and atherosclerosis [3]. Antioxidants may prevent lipid oxidation in cell membrane through inhibition of the attack of free radicals, resulting in increased ability to defend against cellular damage [4]. Thus, taking supplements containing antioxidants is an alternative way of reducing oxidative stress. Since natural antioxidants have an inherent safety advantage, there is increasing interest concerning the use of natural antioxidants to replace synthetic antioxidants. These antioxidants can be separated from many natural products such as herbs $[5,6]$, vegetables [7], and fruits [8]. Mushrooms are also potential sources of antioxidants [9-11], and there is an increasing focus of therapeutic evidence for medicinal mushrooms in their use as anticancer [12-15] and antiviral $[16,17]$ agents.

Tyrosinase is key enzyme of melanogenesis. The antityrosinase activity of mushrooms has also been studied for the developing of functional cosmetics, because the inhibition of tyrosinase can attenuate the synthesis of melanin to have a whitening effect [18]. Antioxidants such as ascorbic acid and its derivatives have been reported to have whitening effect, indicating that melanogenesis could be prevented by the reduction of ROS levels in melanocytes [19]. Tyrosinase is also responsible for the browning of fruits and vegetables [20]. Thus, there has been considerable interest in tyrosinase inhibitors in the food industry and the needs for natural tyrosinase inhibitors have gradually been increased because safety is strictly monitored in the food industry.

Many studies have suggested the antioxidant properties of different mushrooms [21, 22]. These studies have generally used mushroom extracts; little attention has been paid to 
demonstrating the antioxidant activities or antityrosinase activity of mycelium culture supernatants. Recently, secretome analysis has been carried out extensively because the secreted proteins of the mycelium were found to have roles in pathogenesis $[23,24]$. Interestingly, one report focused on the antioxidant activity from mycelium-free broths of Phellinus igniarius [25], and Tsai et al. reported that the spent culture supernatant of Lactobacillus rhamnosus could be used as an attractive source for making cosmetics with antioxidant and antityrosinase activity [26]. These results suggest that mycelium culture media may include ingredients that have antioxidant and antityrosinase activity. Accordingly, we considered that investigating antioxidant and antityrosinase activity from a large number of mycelium culture extracts would be a prerequisite for developing efficient natural antioxidants and tyrosinase inhibitors.

There are more advantages in using the mycelium rather than the fruit body of the mushroom when making products, because the mycelium can easily be produced and is inexpensive compared to cultivating mushrooms. Moreover, using the mycelium could be a more controllable way to develop large-scale products than mushroom cultivation. Consequently, the objective of this study was to determine the potential mycelium culture extracts that have antioxidant and tyrosinase inhibitory properties. Antioxidant activities were determined by testing the scavenging abilities on DPPH, reducing power, and inhibitory power of lipid oxidation. The tyrosinase inhibitory activity of each mycelium culture extract was also examined.

\section{Material and Methods}

2.1. Materials. Ethanol was purchased from Merck (Darmstadt, Germany) and vitamin C, vitamin E, catechin, arbutin, butylated hydroxytoluene (BHT), and DPPH were purchased from Sigma-Aldrich Co. (St. Louis, MO, USA).

\subsection{Mycelium Culture and Preparation of Extract. Mycelium} strains were obtained from National Academy of Agricultural Science in Korea. Each strain was first inoculated to potato dextrose agar plate (PDA) (Difco, Detroit, MI, USA) and the growing $7 \mathrm{~mm}$ size of each mycelium was cut and transferred to $100 \mathrm{~mL}$ of potato dextrose broth (PDB, pH 5.0) (Difco, Detroit, MI, USA). After incubation for 5 days at $25^{\circ} \mathrm{C}$, mycelium of each strain was homogenized and inoculated again to $500 \mathrm{~mL}$ of PDB. Culture media including mycelium were homogenized and extracted with 5 volumes of $40 \%$ ethanol for 72 hours at $25^{\circ} \mathrm{C}$. Extract was then concentrated with rotary evaporator and filtered with 0.45 um membrane filter (Advatec MFS Inc., Japan). Concentrated extract was freeze-dried and used for experiments.

2.3. DPPH Scavenging Activity Assay. DPPH scavenging activity was determined according to the previously described [27]. $0.2 \mathrm{~mL}$ of various concentrations of each EECiM $(10,1,0.1 \mathrm{mg} / \mathrm{mL})$, vitamin C $(0.1 \mathrm{mg} / \mathrm{mL})$, or BHT $(0.1 \mathrm{mg} / \mathrm{mL})$ was added to DPPH solution $(40 \mu \mathrm{M}$, $0.8 \mathrm{~mL}$ ), respectively, and incubated for $10 \mathrm{~min}$ at room temperature. Absorbance at $517 \mathrm{~nm}$ was measured by UV-Vis spectrophotometer (Shimadzu Co. Tokyo, Japan). DPPH scavenging activity (I\%) was calculated as $I \%=\left[1-\left(A_{\text {sample }}-B_{\text {control }}\right) / C_{\text {control }}\right] \times 100$, where $A_{\text {sample }}$ is the absorbance of the sample reaction (containing all reagents and test compound), $B_{\text {control }}$ is the absorbance of the test compound, and $C_{\text {control }}$ is the absorbance of control reaction (containing all reagents without test compound). Vitamin C and BHT were used as positive controls.

2.4. Measuring the Reducing Power. Reducing power of EECiM was measured according to the method of Oyaizu [28]. $0.2 \mathrm{~mL}$ of various concentrations of each $\operatorname{EECiM~}(10,1$, $0.1 \mathrm{mg} / \mathrm{mL})$, vitamin C $(0.1 \mathrm{mg} / \mathrm{mL})$, vitamin $\mathrm{E}(0.1 \mathrm{mg} / \mathrm{mL})$, or BHT $(0.1 \mathrm{mg} / \mathrm{mL})$ was mixed with $0.5 \mathrm{~mL}$ of phosphate buffer $(0.5 \mathrm{M}, \mathrm{pH} 6.0)$ and $0.5 \mathrm{~mL}$ of $1 \%(\mathrm{w} / \mathrm{v})$ potassium ferricyanide $\left[\mathrm{K}_{3} \mathrm{Fe}\left(\mathrm{CN}_{6}\right)\right]$, respectively. After incubating the mixture for $20 \mathrm{~min}$ at $50^{\circ} \mathrm{C}, 0.5 \mathrm{~mL}$ of trichloroacetic acid (TCA) $(10 \% \mathrm{w} / \mathrm{v})$ was added and centrifuged for $10 \mathrm{~min}$ at $1,000 \mathrm{~g}$ (Sigma, Mannheim, Germany). Supernatant $(0.5 \mathrm{~mL})$ was mixed with distilled water $(0.5 \mathrm{~mL})$ and $\mathrm{FeCl}_{3}(0.1 \%$ $\mathrm{w} / \mathrm{v}, 0.5 \mathrm{~mL}$ ). Absorbance at $700 \mathrm{~nm}$ (A700) was measured by using UV-Vis spectrophotometer (Shimadzu. Tokyo, Japan). Vitamin C, Vitamin E, and BHT were used as positive controls.

2.5. Determination of TBA Value. TBA value was determined according to the method of Lin and Chang [29]. $10 \mathrm{mg} / \mathrm{mL}$ of each EECiM $(0.4 \mathrm{~mL})$ and BHT $(0.1 \mathrm{mg} / \mathrm{mL})$ was mixed with $1 \mathrm{~mL}$ of linoleic acid emulsion [linoleic acid solution $(60 \%$ $\mathrm{w} / \mathrm{v}, 0.1 \mathrm{~mL})$, tween $20(0.2 \mathrm{~mL})$, distilled water $(19.7 \mathrm{~mL})]$, $0.5 \mathrm{~mL}$ of $20 \mathrm{mM}$ PBS, and $0.2 \mathrm{~mL}$ of $\mathrm{FeSO}_{4}(0.01 \% \mathrm{w} / \mathrm{v})$. Mixture was then added with $0.2 \mathrm{~mL}$ of $\mathrm{FeSO}_{4}(0.01 \% \mathrm{w} / \mathrm{v})$ and $0.2 \mathrm{~mL}$ of $\mathrm{H}_{2} \mathrm{O}_{2}(0.56 \mathrm{mM})$ and incubated for 6 hours at $37^{\circ} \mathrm{C}$. After incubation, $0.2 \mathrm{~mL}$ of butylated hydroxytoluene (BHT, $0.4 \% \mathrm{w} / \mathrm{v}), 0.2 \mathrm{~mL}$ of trichloroacetic acid ( $4 \% \mathrm{w} / \mathrm{v})$, and $2 \mathrm{~mL}$ of 2-thiobarbituric acid (TBA, $0.8 \% \mathrm{w} / \mathrm{v}$ ) were added and incubated for $30 \mathrm{~min}$ at $100^{\circ} \mathrm{C} .2 \mathrm{~mL}$ of $n$-Butanol was added to mixture and then centrifuged at $200 \mathrm{~g}$ (Sigma, Mannheim, Germany). Absorbance at $532 \mathrm{~nm}$ was measured by using UV-Vis spectrophotometer (Shimadzu, Tokyo, Japan). TBA inhibitory rate $(I \%)$ was calculated: $I \%=\left[1-\left(A_{\text {sample }}-\right.\right.$ $\left.\left.A_{\text {control }}\right) / B_{\text {control }}\right] \times 100$, where $A_{\text {sample }}$ is the absorbance of the sample reaction (containing all reagents and test compound), $A_{\text {control }}$ is the absorbance of the test compound, and $B_{\text {control }}$ is the absorbance of control reaction (containing all reagents without test compound).

2.6. Tyrosinase Inhibitory Activity. Tyrosinase inhibitory activity of each EECiM was determined as described previously [30]. Briefly, mushroom tyrosinase $(20 \mu \mathrm{L}, 1700$ unit/mL) was mixed with $220 \mu \mathrm{L}$ of phosphate buffer $(0.1 \mathrm{M}$, $\mathrm{pH} 6.5)$ and $40 \mu \mathrm{L}$ of $1.5 \mathrm{mM}$ tyrosine and $20 \mu \mathrm{L}$ of different concentrated EECiM $(10,1$, and $0.1 \mathrm{mg} / \mathrm{mL})$. The mixture was then incubated for $15 \mathrm{~min}$ at $37^{\circ} \mathrm{C}$. Following incubation, absorbance of the mixture was determined at $490 \mathrm{~nm}$ by using a UV-Vis spectrophotometer (Shimadzu, Tokyo, Japan). Arbutin $(10,1$, and $0.1 \mathrm{mg} / \mathrm{mL})$ and vitamin $\mathrm{C}(10 \mathrm{mg} / \mathrm{mL})$ 
were used as positive control in this study. Percent inhibition of tyrosinase activity was determined according to the formula: inhibition $(\%)=100-\left(W_{\text {sample }} / W_{\text {blank }}\right) \times 100$, where $W$ is the absorbance at $490 \mathrm{~nm}$. $W_{\text {blank }}$ is the absorbance of control reaction (containing all reagents without test compound).

\section{Results}

3.1. Preparation of EECiMs. Fifty-five EECiMs were prepared according to the method described in Section 2. Each EECiM was used for measuring the antioxidant activities and tyrosinase inhibitory properties. As shown in Figures 1-4, several EECiMs showed strong antioxidant activities and tyrosinase inhibitory property indicated that mycelium including culture media could be a useful source for preparing the natural antioxidant.

\subsection{Several EECiMs Showed Strong DPPH Scavenging Activity.} The DPPH assay is a well-known method that is used to determine antioxidant properties, because DPPH can donate hydrogen when DPPH reacts with an antioxidant, resulting in a change in color. Thus, the antioxidant activities of each EECiM were first screened by measuring the DPPH scavenging ability. Most EECiM showed low or negligible DPPH scavenging activity (data not shown) but EECiM containing MEVE, APO, TAU, VBO, and Osp showed relatively high DPPH scavenging activity (Figure 1 and Table 1). Although those DPPH scavenging activities were lower than that of vitamin C $(94.3 \pm 0.0 \%$ at a concentration of $0.1 \mathrm{mg} / \mathrm{mL})$ and BHT $(82.2 \pm 0.0 \%$ at a concentration of $0.1 \mathrm{mg} / \mathrm{mL})$, the EECiM of APO showed the highest DPPH scavenging activity $(77.4 \pm 4.4 \%)$ at a concentration of $10 \mathrm{mg} / \mathrm{mL}$. Other EECiMs such as MEVE (43.7 $\pm 1.9 \%)$, TAU ( $42.6 \pm 1.1 \%)$, and VBO $(37.5 \pm 5.4 \%)$ also showed DPPH scavenging activities at a concentration of $10 \mathrm{mg} / \mathrm{mL}$. These data suggest that the five EECiMs that were found in this study have good potential as antioxidants.

3.3. APO EECiM Showed Highest Reducing Power. Since the antioxidant action was performed by reductones, which is associated with reducing power through the breakage of the free radical chain, we next investigated the reducing power of five EECiMs, which found at DPPH scavenging test, at various concentrations for each EECiM $(0.1,1$, and $10 \mathrm{mg} / \mathrm{mL})$, vitamin $C(0.1 \mathrm{mg} / \mathrm{mL})$, vitamin $E(0.1 \mathrm{mg} / \mathrm{mL})$, and BHT $(0.1 \mathrm{mg} / \mathrm{mL})$. As shown in Figure 2, all five EECiMs apparently exhibited reducing power that increased in a dosedependent manner. The reducing powers (A700) of the APO EECiM, which showed the highest reducing power from among the five EECiMs, were $0.14 \pm 0.002,0.24 \pm 0.003$, and $1.18 \pm 0.041$ at concentrations of $0.1,1$, and $10 \mathrm{mg} / \mathrm{mL}$, respectively. Meanwhile, the reducing powers (A700) of vitamin C, vitamin $\mathrm{E}$, and $\mathrm{BHT}$ at a concentration of $0.1 \mathrm{mg} / \mathrm{mL}$ were $0.85 \pm 0.019,0.64 \pm 0.005$, and $0.72 \pm 0.003$, respectively.

3.4. EECiMs Showed Inhibitory Activity of Lipid Oxidation. Since thiobarbituric acid (TBA) can form a complex with

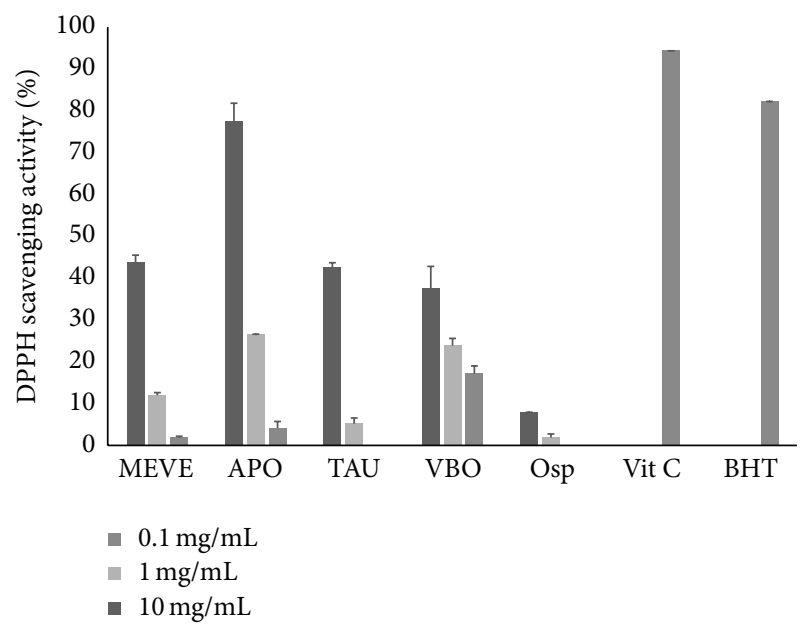

FIgURE 1: DPPH radical scavenging activities of five EECiM. Each EECiM at various concentrations $(0.1,1,10 \mathrm{mg} / \mathrm{mL})$, vitamin $\mathrm{C}$ $(0.1 \mathrm{mg} / \mathrm{mL})$, and BHT $(0.1 \mathrm{mg} / \mathrm{mL})$ was interacted with DPPH. Values represented as means $\pm \mathrm{SD}(n=3)$. MEVE, APO, TAU, VBO, and Osp represent the EECiM of Morchella esculenta var. esculenta, Auricularia polytricha, Tremella aurantia, Volvariella bombycina, and Oudemansiella sp., respectively.

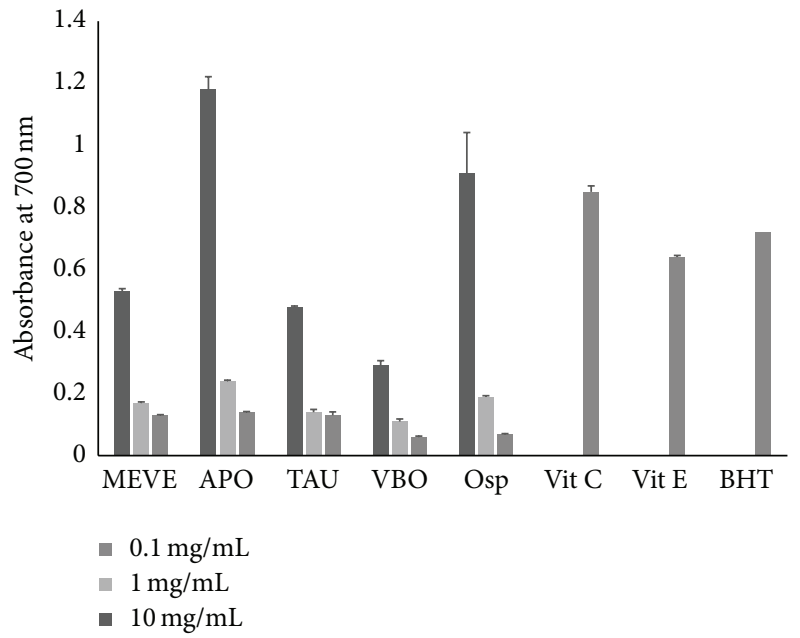

FIGURE 2: Reducing power of five EECiMs. Different concentrations of five EECiMs $(0.1,1$, and $10 \mathrm{mg} / \mathrm{mL})$, vitamin C $(0.1 \mathrm{mg} / \mathrm{mL})$, vitamin E $(0.1 \mathrm{mg} / \mathrm{mL})$, BHT $(0.1 \mathrm{mg} / \mathrm{mL})$ were used for investigating the reducing power. Results are represented as absorbance at $700 \mathrm{~nm}(\mathrm{~A} 700)$ and values are expressed as means $\pm \mathrm{SD}(n=3)$. MEVE, APO, TAU, VBO, and Osp represent the EECiM of Morchella esculenta var. esculenta, Auricularia polytricha, Tremella aurantia, Volvariella bombycina, and Oudemansiella sp., respectively.

malondialdehyde (MDA), which is a major product of oxidized lipid materials, the TBA-MDA complex has been used to determine the degree of lipid oxidation in biological tissue or food [31]. To evaluate the inhibitory effect of EECiMs during lipid oxidation, the TBA value was determined by adding each EECiM, which found by DPPH scavenging test, to the linoleic acid peroxidation reaction. As shown in Figure 3, five EECiMs showed a strong inhibitory effect 


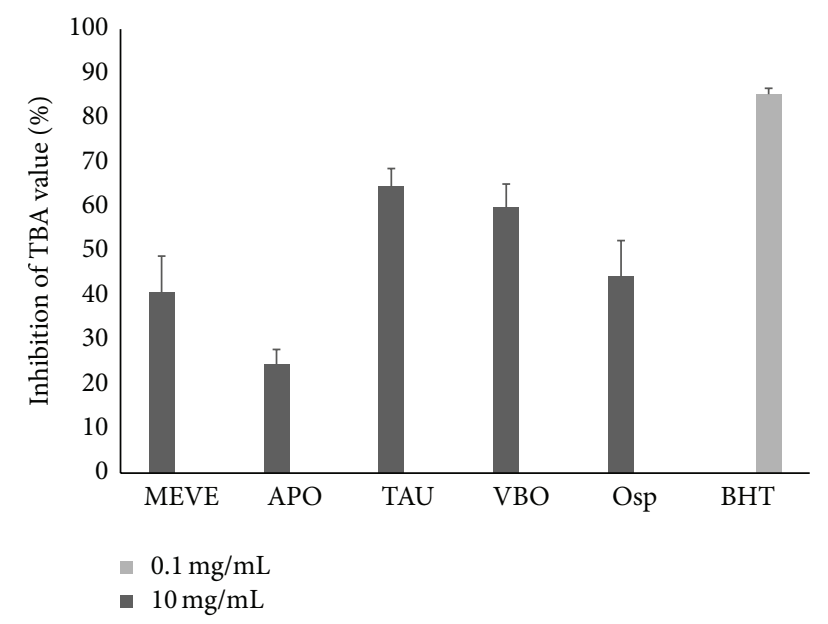

FIgUre 3: Inhibitory activity of TBA values of five EECiMs. $10 \mathrm{mg} / \mathrm{mL}$ of each EECiM and $0.1 \mathrm{mg} / \mathrm{mL}$ of BHT were used for determining the inhibitory activity of TBA value. Results are represented as inhibitory rate (\%) of TBA value and expressed as means $\pm \mathrm{SD}(n=3)$. MEVE, APO, TAU, VBO, and Osp represent the EECiM of Morchella esculenta var. esculenta, Auricularia polytricha, Tremella aurantia, Volvariella bombycina, and Oudemansiella sp., respectively.

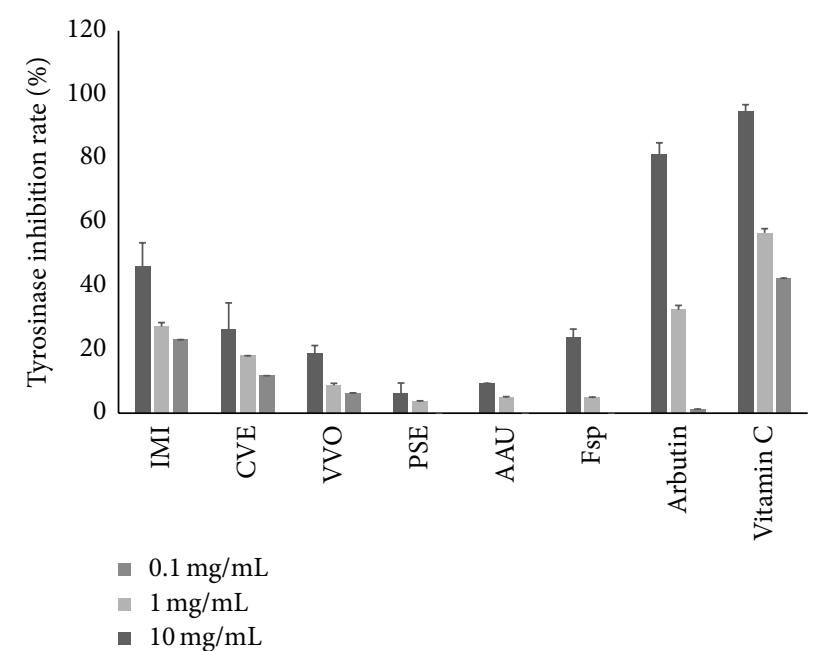

Figure 4: Tyrosinase inhibitory activities of EECiMs. Various concentrations $(0.1,1$, and $10 \mathrm{mg} / \mathrm{mL})$ of EECiM and arbutin were used for determining the tyrosinase inhibitory activity. Arbutin and vitamin $\mathrm{C}$ were used as positive control for measuring the tyrosinase inhibitory activity. Values represented as means \pm SD $(n=3)$. IMI, CVE, VVO, PSE, AAU, and Fsp represent the EECiM of Inonotus mikadoi, Coriolus versicolor, Volvariella volvacea, Panellus serotinus, Auricularia auricular, and Fomitopsis sp., respectively.

on linoleic acid peroxidation ranging from $24.5 \%$ to $64.5 \%$. Interestingly, the APO EECiM, which showed the highest DPPH scavenging activity and reducing power (Figures 1 and 2), was shown to have the lowest inhibitory activity during linoleic acid peroxidation $(24.5 \pm 3.3 \%)$ from among the five EECiMs (Figure 3). These data indicate that the five EECiMs, which showed in Figure 3, have inhibitory potential
TABLE 1: Mycelium strains which are used in this study.

\begin{tabular}{|c|c|c|}
\hline & Genus & Species \\
\hline 1 & \multirow{4}{*}{ Auricularia } & sp. \\
\hline 2 & & auricula-judae \\
\hline 3 & & polytricha \\
\hline 4 & & auricula \\
\hline 5 & Cerrena & unicolor \\
\hline 6 & \multirow{2}{*}{ Clitocybe } & maxima \\
\hline 7 & & aurantiaca \\
\hline 8 & \multirow{5}{*}{ Collybia } & abundans \\
\hline 9 & & peronata \\
\hline 10 & & confluens \\
\hline 11 & & erythropus \\
\hline 12 & & aquosa \\
\hline 13 & Mycena & pelianthina \\
\hline 14 & \multirow{2}{*}{ Coprinus } & cinereus \\
\hline 15 & & comatus \\
\hline 16 & \multirow{3}{*}{ Coriolus } & brevis \\
\hline 17 & & hirsutus \\
\hline 18 & & versicolor \\
\hline 19 & Dacrymyces & palmatus \\
\hline 20 & \multirow{2}{*}{ Fomes } & sp. \\
\hline 21 & & fomentarius \\
\hline 22 & Fomitella & fraxinea \\
\hline 23 & \multirow{4}{*}{ Fomitopsis } & roseus \\
\hline 24 & & pubertasis \\
\hline 25 & & sp. \\
\hline 26 & & pinicola \\
\hline 27 & Hericium & erinaceus \\
\hline 28 & \multirow{3}{*}{ Hohenbuehelia } & petaloides \\
\hline 29 & & sp. \\
\hline 30 & & myxotricha \\
\hline 31 & \multirow{4}{*}{ Inonotus } & xeranticus \\
\hline 32 & & hispidus \\
\hline 33 & & obliqua \\
\hline 34 & & mikadoi \\
\hline 35 & Macrolepiota & alborubescens \\
\hline 36 & \multirow{4}{*}{ Morchella } & esculenta var.umbrina \\
\hline 37 & & esculenta var.esculenta \\
\hline 38 & & esculenta \\
\hline 39 & & elata \\
\hline 40 & \multirow{5}{*}{ Oudemansiella } & mucida \\
\hline 41 & & radicata \\
\hline 42 & & sp. \\
\hline 43 & & pudens \\
\hline 44 & & brunneomarginata \\
\hline 45 & \multirow{3}{*}{ Panellus } & serotinus \\
\hline 46 & & stipticus \\
\hline 47 & & sp. \\
\hline 48 & Phyllotopsis & nidulans \\
\hline
\end{tabular}


TABLE 1: Continued.

\begin{tabular}{lcc}
\hline & Genus & Species \\
\hline 49 & Rhodotus & palmatus \\
\hline 50 & Wolfiporia & cocos \\
\hline 51 & Schizophyllum & commune \\
\hline 52 & Tremella & sp. \\
53 & & aurantia \\
\hline 54 & Volvariella & bombycina \\
55 & & volvacea \\
\hline
\end{tabular}

during lipid oxidation and could be used to develop antilipid oxidants.

3.5. Antityrosinase Activity Was Detected in EECiMs. Since the tyrosinase has an important role in melanin synthesis, the materials which have an inhibitory activity of tyrosinase are considered as good candidate for getting whitening effect. So, we next investigated the tyrosinase inhibitory potentials of 55 EECiMs at various concentrations for each EECiM (0.1, 1, and $10 \mathrm{mg} / \mathrm{mL})$, arbutin $(0.1,1$, and $10 \mathrm{mg} / \mathrm{mL})$, and vitamin C $(0.1,1$, and $10 \mathrm{mg} / \mathrm{mL})$. Interestingly, EECiMs which showed high antioxidant properties (Figures 1, 2, and 3) exhibited very low tyrosinase inhibitory activity $(<3 \%$ at $10 \mathrm{mg} / \mathrm{mL}$ concentration). Relatively high tyrosinase inhibitory activity was detected from EECiMs containing IMI (46.0 $\pm 7.5 \%)$, CVE $(26.3 \pm 8.3 \%)$, VVO $(18.8 \pm 2.5 \%)$, PSE $(6.3 \pm 3.2 \%)$, AAU $(9.4 \pm 0.0 \%)$, and Fsp $(23.9 \pm 2.5 \%)$ at the concentration of $10 \mathrm{mg} / \mathrm{mL}$ (Figure 4 ).

\section{Discussion}

This study examined the antioxidant properties and antityrosinase activity of 55 different mycelium culture extracts. Among them, five EECiMs of MEVE, APO, TAU, VBO, and Osp were selected due to their reliable antioxidant properties, and EECiMs of IMI, CVE, VVO, PSE, AAU, and Fsp were determined to have relatively high tyrosinase inhibitory activity. Although our study proposed a preliminarily investigation of antioxidant and antityrosinase activity, to our knowledge, this is the first report demonstrating those properties with each mycelium culture extract. Since the use of mycelium culture extracts is advantageous in large-scale production, we considered that a preliminary investigation of medicinal properties such as antioxidant and antityrosinase activity was required for a large number of different strains. Although the actual components which represent these properties could not be identified in this study, if we consider that most papers have focused on analyzing one or two strains to elucidate their antioxidant property, our study contributes significant information about EECiM candidates that have strong antioxidant or antityrosinase activity.

Until now, research on the medicinal properties of APOwhich showed the highest DPPH scavenger activity in this study (Figure 1)-seems to have mostly focused on its anticancer effects [32]. Although the antihypercholesterolemic effect of APO was recently reported [33], little attention has been paid to the antioxidant property of APO culture extract.
In addition, the EECiM of APO was revealed to have strong reducing power (Figure 2), as well as TBA inhibitory activity (Figure 3). Because mycelium culture in liquid medium has an advantage in quantitative biomass production, our study strongly suggests that EECiM of APO has an advantage in developing natural antioxidants.

In the case of TAU, only a few studies have demonstrated its effect on glucose metabolism, including its antidiabetic effect [33]. TAU polysaccharide seems to have critical roles in regulating the enzyme activity related with glucose metabolisms [34]. In our study, EECiM of TAU showed antioxidant activity. Interestingly, EECiM of TAU revealed the highest inhibitory effect on lipid oxidation (Figure 3). Functional analysis of TAU polysaccharide on lipid oxidation will give us a deeper understanding of the regulatory role of EECiM of TAU in lipid oxidation.

Tyrosinase is a key enzyme of melanogenesis and is also called polyphenol oxidase. Since tyrosinase inhibitors can be used as effective skin whitening agents, many natural sources such as grape seed and raspberry have been studied to investigate their antityrosinase activity [35]. Although the actual components that have antityrosinase activity were not identified in this study, we found several attractive EECiM candidates which could be used as natural tyrosinase inhibitors. Among them, EECiM containing IMI showed the highest tyrosinase inhibitory property among the 55 different EECiMs, and about $45 \%$ of tyrosinase activity was inhibited at a concentration of $10 \mathrm{mg} / \mathrm{mL}$. Although one report detected lipoxygenase inhibitory activity from IMI [36], IMI is a rarely characterized species. In our study, EECiMs of IMI and CVE showed high tyrosinase inhibitory activity (Figure 4). Interestingly, EECiM of IMI showed the highest astringency effect among 55 different EECiMs, and CVE also showed a high astringency effect (data not shown). Taken together, these data strongly suggested that EECiMs that are selected to have tyrosinase inhibitory activity could be developed as functional additives for cosmetics.

Consequently, our study proposed several candidates for natural antioxidants and tyrosinase inhibitors. Although we found that vitamin $\mathrm{C}$ has strong antioxidant and antityrosinase activities (Figures 1, 2, and 4), we could not find EECiMs which have strong both antioxidant and antityrosinase activities. This fact indicated that each EECiM possesses different components to show antioxidant and antityrosinase properties. The in vitro antioxidant activity of mushroom extracts seems to be closely correlated with its polysaccharide contents [37] or phenolic components [38]. Several studies have suggested natural tyrosinase inhibitors such as flavonoids [39] and phloroglucinol derivatives [40]. Analysis of active components of the EECiMs selected in this study will give us a deeper understanding of the mechanisms of antioxidant and tyrosinase inhibition of EECiMs. Fractionation to isolate the active components of EECiMs and further identification are in progress.

\section{Conclusion}

In this study, we found the several mycelia which have DPPH radical scavenging activity, reducing power, inhibitory 
activity of TBA value, and tyrosinase inhibition. In addition, we included the culture media of each mycelium in making extracts and showed strong antioxidant activities and a tyrosinase inhibitory property, which indicated that EECiM is a useful source for making antioxidants. Thus, the EECiMs that were determined to have antioxidant activities and a tyrosinase inhibitory property in our study can be used as additives in natural antioxidants and functional cosmetics.

\section{Conflict of Interests}

The authors declare that there is no conflict of interests regarding the publication of this paper.

\section{Authors' Contribution}

Ki Moon Park and Kyung Min Kwon have equally contributed to this work.

\section{Acknowledgment}

This work was supported by an Incheon National University Research Grant in 2012.

\section{References}

[1] A. Takaki and K. Yamamoto, "Control of oxidative stress in hepatocellular carcinoma: helpful or harmful?" World Journal of Hepatology, vol. 7, no. 7, pp. 968-979, 2015.

[2] M. M. Briehl, "Oxygen in human health from life to death-an approach to teaching redox biology and signaling to graduate and medical students," Redox Biology, vol. 5, pp. 124-139, 2015.

[3] D. C. Ellinsworth, "Arsenic, reactive oxygen, and endothelial dysfunction," Journal of Pharmacology and Experimental Therapeutics, vol. 353, no. 3, pp. 458-464, 2015.

[4] B.-S. Wang, L.-W. Chang, W.-J. Yen, and P.-D. Duh, "Antioxidative effect of sesame coat on LDL oxidation and oxidative stress in macrophages," Food Chemistry, vol. 102, no. 1, pp. 351-360, 2007.

[5] A. Kasrati, C. A. Jamali, K. Bekkouche, H. Wohlmuth, D. Leach, and A. Abbad, "Comparative evaluation of antioxidant and insecticidal properties of essential oils from five Moroccan aromatic herbs," Journal of Food Science and Technology, vol. 52, no. 4, pp. 2312-2319, 2015.

[6] Y.-C. Zhang, G. Li, C. Jiang et al., "Tissue-specific distribution of ginsenosides in different aged ginseng and antioxidant activity of ginseng leaf," Molecules, vol. 19, no. 11, pp. 17381-17399, 2014.

[7] S. S. Umesha and K. A. Naidu, "Antioxidants and antioxidant enzymes status of rats fed on n-3 PUFA rich Garden cress (Lepidium SativumL) seed oil and its blended oils," Journal of Food Science and Technology, vol. 52, no. 4, pp. 1993-2002, 2015.

[8] B. Abdennacer, M. Karim, M. Yassine, R. Nesrine, D. Mouna, and B. Mohamed, "Determination of phytochemicals and antioxidant activity of methanol extracts obtained from the fruit and leaves of Tunisian Lycium intricatum Boiss.", Food Chemistry, vol. 174, pp. 577-584, 2015.

[9] H. Li, H.-S. Lee, S.-H. Kim, B. Moon, and C. Lee, "Antioxidant and anti-inflammatory activities of methanol extracts of Tremella fuciformis and its major phenolic acids," Journal of Food Science, vol. 79, no. 4, pp. C460-C468, 2014.
[10] F. Kalyoncu, M. Oskay, H. Sağlam, T. F. Erdoğan, and A. U. Tamer, "Antimicrobial and antioxidant activities of mycelia of 10 wild mushroom species," Journal of Medicinal Food, vol. 13, no. 2, pp. 415-419, 2010.

[11] J. Cilerdzic, M. Stajic, J. Vukojevic, I. Milovanovic, and N. Muzgonja, "Antioxidant and antifungal potential of Pleurotus ostreatus and Agrocybe cylindracea basidiocarps and mycelia," Current Pharmaceutical Biotechnology, vol. 16, no. 2, pp. 179186, 2015.

[12] J. Loganathan, J. Jiang, A. Smith et al., "The mushroom Ganoderma lucidum suppresses breast-to-lung cancer metastasis through the inhibition of pro-invasive genes," International Journal of Oncology, vol. 44, no. 6, pp. 2009-2015, 2014.

[13] A. Rouhana-Toubi, S. P. Wasser, A. Agbarya, and F. Fares, "Inhibitory effect of ethyl acetate extract of the shaggy inc cap medicinal mushroom, Coprinus comatus (Higher Basidiomycetes) fruit bodies on cell growth of human ovarian cancer," International Journal of Medicinal Mushrooms, vol. 15, no. 5, pp. 457-470, 2013.

[14] I. M. W. Ruma, E. W. Putranto, E. Kondo et al., "Extract of Cordyceps militaris inhibits angiogenesis and suppresses tumor growth of human malignant melanoma cells," International Journal of Oncology, vol. 45, no. 1, pp. 209-218, 2014.

[15] K.-W. Luo, G. G.-L. Yue, C.-H. Ko et al., "In vivo and in vitro antitumor and anti-metastasis effects of Coriolus versicolor aqueous extract on mouse mammary 4T1 carcinoma," Phytomedicine, vol. 21, no. 8-9, pp. 1078-1087, 2014.

[16] C. R. Wang, R. Zhou, T. B. Ng, J. H. Wong, W. T. Qiao, and F. Liu, "First report on isolation of methyl gallate with antioxidant, anti-HIV-1 and HIV-1 enzyme inhibitory activities from a mushroom (Pholiota adiposa)," Environmental Toxicology and Pharmacology, vol. 37, no. 2, pp. 626-637, 2014.

[17] S. Lee, J. I. Kim, J. Heo et al., "The anti-influenza virus effect of Phellinus igniarius extract," Journal of Microbiology, vol. 51, no. 5, pp. 676-681, 2013.

[18] S. Baek, I. Nam, H. Kwak, K. Kim, and S. Lee, "Cellular anti-melanogenic effects of a euryale ferox seed extract ethyl acetate fraction via the lysosomal degradation machinery," International Journal of Molecular Sciences, vol. 16, no. 5, pp. 9217-9235, 2015.

[19] A. Balaguer, A. Chisvert, and A. Salvador, "Environmentally friendly LC for the simultaneous determination of ascorbic acid and its derivatives in skin-whitening cosmetics," Journal of Separation Science, vol. 31, no. 2, pp. 229-236, 2008.

[20] J. S. Roh, J. Y. Han, J. H. Kim, and J. K. Hwang, "Inhibitory effects of active compounds isolated from safflower (Carthamus tinctorius L.) seeds for melanogenesis," Biological and Pharmaceutical Bulletin, vol. 27, no. 12, pp. 1976-1978, 2004.

[21] S. J. Huang, C. P. Lin, J. L. Mau, Y. S. Li, and S. Y. Tsai, "Effect of UV-B irradiation on physiologically active substance content and antioxidant properties of the medicinal caterpillar fungus Cordyceps militaris (ascomycetes)," International Journal of Medicinal Mushrooms, vol. 17, no. 3, pp. 241-253, 2015.

[22] Y.-S. Tan, A. Baskaran, N. Nallathamby, K.-H. Chua, U. R. Kuppusamy, and V. Sabaratnam, "Influence of customized cooking methods on the phenolic contents and antioxidant activities of selected species of oyster mushrooms (Pleurotus spp.)," Journal of Food Science and Technology, vol. 52, no. 5, pp. 3058-3064, 2015.

[23] S. S. Weber, A. F. A. Parente, C. L. Borges, J. A. Parente, A. M. Bailão, and C. M. D. A. Soares, "Analysis of the secretomes of 
Paracoccidioides mycelia and yeast cells," PLoS ONE, vol. 7, no. 12, Article ID e52470, 2012.

[24] R. González-Fernández, K. Aloria, J. Valero-Galván, I. Redondo, J. M. Arizmendi, and J. V. Jorrín-Novo, "Proteomic analysis of mycelium and secretome of different Botrytis cinerea wild-type strains," Journal of Proteomics, vol. 97, pp. 195-221, 2014.

[25] M. Y. Lung, J. C. Tsai, and P. C. Huang, "Antioxidant properties of edible basidiomycete phellinus igniarius in submerged cultures," Journal of Food Science, vol. 75, no. 1, pp. 18-24, 2010.

[26] C.-C. Tsai, C.-F. Chan, W.-Y. Huang et al., "Applications of Lactobacillus rhamnosus spent culture supernatant in cosmetic antioxidation, whitening and moisture retention applications," Molecules, vol. 18, no. 11, pp. 14161-14171, 2013.

[27] C. Sánchez-Moreno, J. A. Larrauri, and F. Saura-Calixto, "A procedure to measure the antiradical efficiency of polyphenols," Journal of the Science of Food and Agriculture, vol. 76, no. 2, pp. 270-276, 1998.

[28] M. Oyaizu, "Studies on products of browning reaction. Antioxidative activities of products of browning reaction prepared from glucosamine," The Japanese Journal of Nutrition and Dietetics, vol. 44, no. 6, pp. 307-315, 1986.

[29] M.-Y. Lin and F.-J. Chang, "Antioxidative effect of intestinal bacteria Bifidobacterium longum ATCC 15708 and Lactobacillus acidophilus ATCC 4356," Digestive Diseases and Sciences, vol. 45, no. 8, pp. 1617-1622, 2000.

[30] Y. Ishihara, M. Oka, M. Tsunakawa et al., "Melanostatin, a new melanin synthesis inhibitor. Production, isolation, chemical properties, structure and biological activity," The Journal of Antibiotics, vol. 44, no. 1, pp. 25-32, 1991.

[31] B. Tokur, K. Korkmaz, and D. Ayas, "Comparison of two Thiobarbituric Acid (TBA) method for monitoring lipid oxidation in fish," E.U. Journal of Fisheries \& Aquatic Sciences, vol. 23, pp. 331-334, 2006.

[32] J. Yu, R. Sun, Z. Zhao, and Y. Wang, "Auricularia polytricha polysaccharides induce cell cycle arrest and apoptosis in human lung cancer A549 cells," International Journal of Biological Macromolecules, vol. 68, pp. 67-71, 2014.

[33] S. Zhao, C. Rong, Y. Liu et al., "Extraction of a soluble polysaccharide from Auricularia polytricha and evaluation of its antihypercholesterolemic effect in rats," Carbohydrate Polymers, vol. 122, pp. 39-45, 2015.

[34] T. Kiho, H. Morimoto, T. Kobayashi et al., "Effect of a polysaccharide (TAP) from the fruiting bodies of Tremella aurantia on glucose metabolism in mouse liver," Bioscience, Biotechnology and Biochemistry, vol. 64, no. 2, pp. 417-419, 2000.

[35] N. Smit, J. Vicanova, and S. Pavel, "The hunt for natural skin whitening agents," International Journal of Molecular Sciences, vol. 10, no. 12, pp. 5326-5349, 2009.

[36] S. W. Lee, J.-G. Song, B. S. Hwang et al., "Lipoxygenase inhibitory activity of Korean indigenous mushroom extracts and isolation of an active compound from Phellinus baumii," Mycobiology, vol. 42, no. 2, pp. 185-188, 2014.

[37] P. Chen, Y. Yong, Y. Gu, Z. Wang, S. Zhang, and L. Lu, "Comparison of antioxidant and antiproliferation activities of polysaccharides from eight species of medicinal mushrooms," International Journal of Medicinal Mushrooms, vol. 17, no. 3, pp. 287-295, 2015.

[38] W. Radzki, A. Sławińska, E. Jabłońska-Ryś, and W. Gustaw, "Antioxidant capacity and polyphenolic content of dried wild edible mushrooms from Poland," International Journal of Medicinal Mushrooms, vol. 16, no. 1, pp. 65-75, 2014.
[39] K. Shimizu, R. Kondo, and K. Sakai, "Inhibition of tyrosinase by flavonoids, stilbenes and related 4- substituted resorcinols: Structure-activity investigations," Planta Medica, vol. 66, no. 1, pp. 11-15, 2000.

[40] H. S. Kang, H. R. Kim, D. S. Byun, B. W. Son, T. J. Nam, and J. S. Choi, "Tyrosinase inhibitors isolated from the edible brown alga Ecklonia stolonifera," Archives of Pharmacal Research, vol. 27, no. 12, pp. 1226-1232, 2004. 


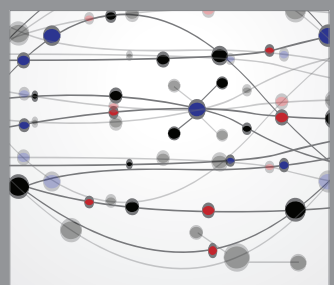

The Scientific World Journal
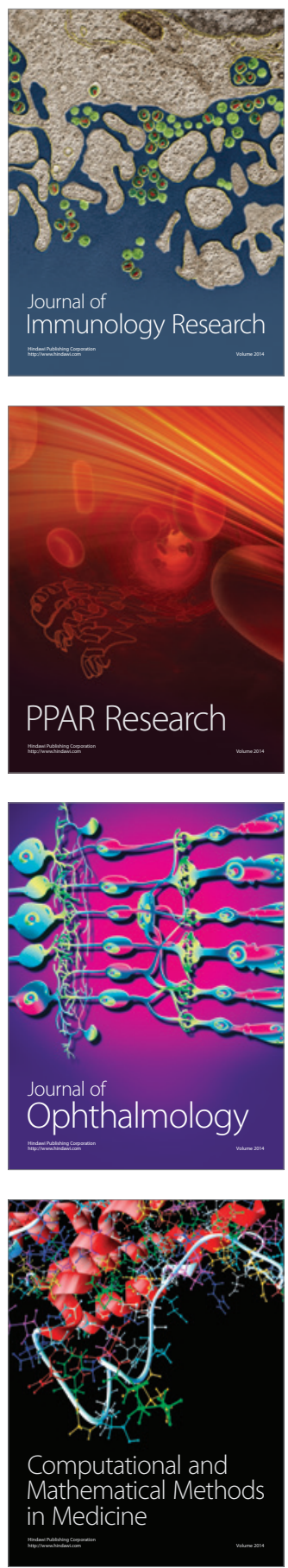

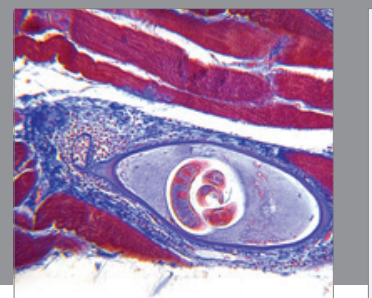

Gastroenterology

Research and Practice
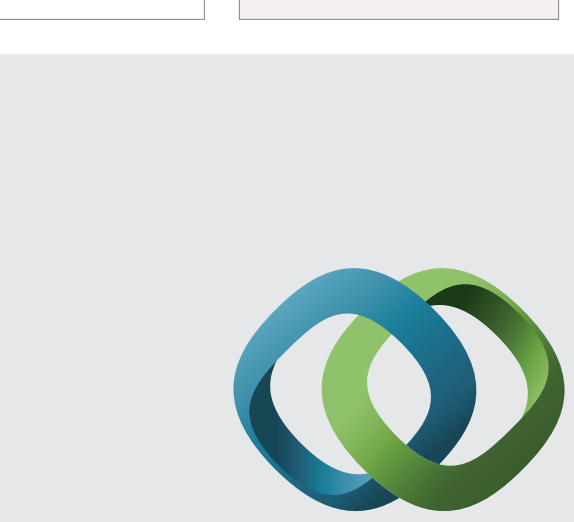

\section{Hindawi}

Submit your manuscripts at

http://www.hindawi.com
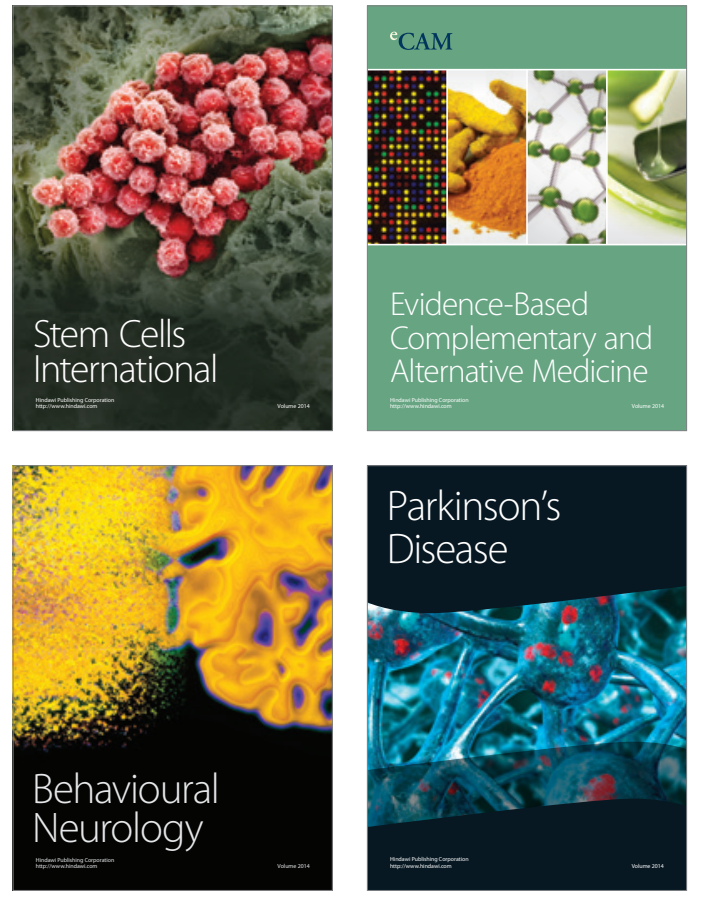
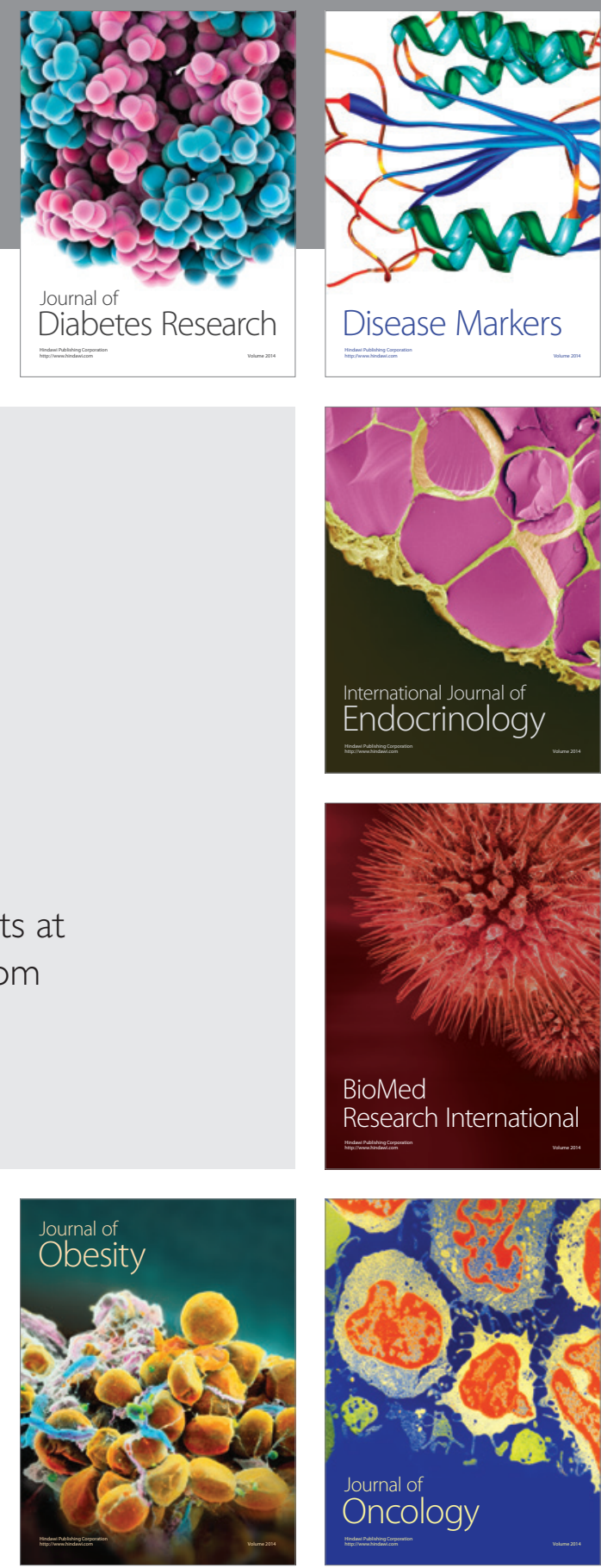

Disease Markers
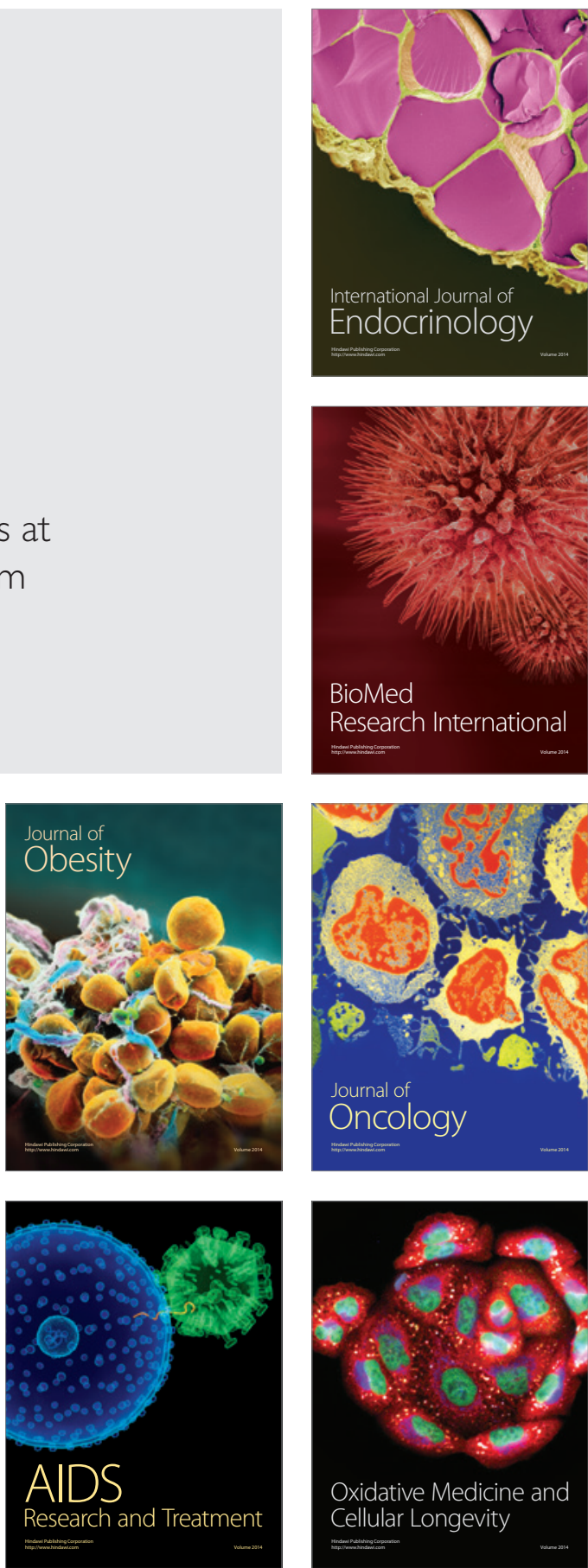